\title{
Achievement of Surgical Proficiency in Laparoscopic Surgery for Early Endometrial Cancer by Gynecologic Oncologists
}

\author{
KOUICHIRO KAWANO, NAOTAKE TSUDA, ATSUMU TERADA, JUNGMYUNG PARK, \\ HIROKI NASU, KEN MATSUKUMA, SHIN NISHIO AND KIMIO USHIJIMA \\ Department of Obstetrics and Gynecology, Kurume University School of Medicine, Kurume 830-0011, Japan \\ Received 12 December 2018, accepted 26 February 2019 \\ J-STAGE advance publication 1 May 2020
}

Edited by TOSHIYUKI YOSHIZATO

\begin{abstract}
Summary: Objective: Although studies have evaluated learning curves for laparoscopic surgery (LS), this issue has not yet been addressed in gynecologic oncologists. The present study aimed to evaluate LS proficiency for early-stage endometrial cancer among gynecologic oncologists.

Methods: We examined 25 cases in which LS with pelvic lymphadenectomy (PLA) for endometrial cancer was performed by two gynecologic oncologists undergoing training in LS. The LS duration, estimated blood loss (EBL), number of dissected pelvic lymph nodes (PLNs), and perioperative complications were assessed as measures of surgical proficiency.

Results: Operators A and B performed 10 and 15 cases, respectively, with median LS durations of 348.5 and 378 minutes, respectively. Although the LS duration and number of procedures did not exhibit a significant correlation, the regression lines for both operators showed decreasing trends. Both operators had a consistently low median EBL (A, $57 \mathrm{ml}$; B, $60 \mathrm{ml}$ ). Operators A and B dissected a median of 21.5 and 22 PLNs, respectively. Although both operators dissected a relatively large number of PLNs, with a median of over 20 per patient after the introduction of LS, this number decreased as the number of LS increased for Operator $B(p=0.009)$. However, no correlation was observed between the LS duration and number of PLNs dissected by Operator B $(\rho=0.353, p=0.197)$. A severe perioperative complication, specifically perforation of the sigmoid colon requiring emergent laparotomy, occurred in one case; however, the association with surgical manipulation was not definitive.
\end{abstract}

Conclusion: The observed gynecologic oncologists were able to acquire early proficiency in LS for early-stage endometrial cancer.

Key words laparoscopic surgery, endometrial cancer, gynecologic oncologist, learning curve, safety

\section{INTRODUCTION}

Endometrial cancer (EC) is the most common gynecologic cancer in Japan, with approximately 13,800 cases reported in 2014 [1,2]. EC is mainly treated via surgery, and recent trends have favored the use of minimally invasive surgery, which is associated with reduced postoperative pain, earlier recovery, and an improved quality of life. For example, in the United States, the frequency of minimally invasive laparo- scopic surgeries (LS) for EC increased from $9.3 \%$ in 2006 to $61.7 \%$ in 2011 [3]. Accordingly, several randomized controlled studies have compared and verified the safety (4-6) and prognosis [7] associated with LS and open surgery for EC. Several reports have described the LS learning curve for EC but have not focused on gynecologic oncologists. This study aimed to evaluate the proficiency in LS for early-stage EC by gynecologic oncologists with considerable experience

Corresponding Author: Kouichiro Kawano, M.D., Ph.D., Department of Obstetrics and Gynecology, Kurume University School of Medicine, 67 Asahimachi, Kurume, Fukuoka 830-0011, Japan. Tel: +81-942-31-7573, Fax: +81-942-35-0238, E-mail: kawano_kouichirou@kurume-u.ac.jp 
in laparotomy procedures for EC.

\section{MATERIALS AND METHODS}

\section{Patients and operators}

This retrospective study consisted of cases in which LS with pelvic lymphadenectomy (PLA) was performed for the treatment of EC at Kurume University Hospital between February 2015 and October 2017. The institutional review board approved the study protocol (No. 17222).

The indications of LS for EC were as follows: clinical stage IA, endometrioid carcinoma grade 1 or 2 in preoperative histology, and a uterine body smaller than a fist. Patients with a body mass index (BMI) of $\geq 35 \mathrm{~kg} / \mathrm{m}^{2}$ or with preoperatively predicted severe pelvic adhesions were excluded. All procedures were performed by two gynecologic oncologists (Operators $\mathrm{A}$ and $\mathrm{B}$ ) undergoing training in LS.

\section{Procedure of LS for endometrial cancer}

All patients underwent an extended simple hysterectomy, which corresponded to the type II radical hysterectomy described in the revised Piver classification [8]. The external uterine os was sutured to avoid tumor leakage prior to the abdominal procedure. Initially, a $12-\mathrm{mm}$ port was inserted through the umbilicus to enable insertion of a 10-mm video scope. Pneumoperitoneum was generated using $\mathrm{CO}_{2}$ to achieve an intraabdominal pressure of $10 \mathrm{mmHg}$. After inspecting the abdominal cavity, three ports were inserted in a modified diamond configuration: two 5-mm ports were placed $2 \mathrm{~cm}$ above and $2 \mathrm{~cm}$ medial to both sides of the superior anterior iliac spine, while a $12-\mathrm{mm}$ port was placed between the pubic symphysis and umbilicus. A uterine manipulator was not used. A VagiPipe ${ }^{\mathrm{TM}}$ (Hakko Co. Ltd, Chikuma, Nagano, Japan) was then introduced vaginally to identify the vaginal fornix. Hysterectomy was performed using Ligasure ${ }^{\mathrm{TM}}$ Maryland (Coviden, Dublin, Ireland), monopolar and bipolar energy devices after sealing the fallopian tubes with Ligasure ${ }^{\mathrm{TM}}$. After the uterine artery was separated from the ureter and ligated, the cardinal ligament was transected between the uterine artery and ureter without opening the ureter tunnel. The uterus was then placed in a Memobag ${ }^{\mathrm{TM}}$ and retrieved through the vagina. The external iliac, inguinal, obturator, internal iliac, and common iliac lymph nodes were dissected via PLA, and the pelvic lymph nodes (PLNs) were retrieved through a reducer sleeve to avoid port site metastasis.

\section{Evaluation of surgical proficiency}

Surgical proficiency was evaluated using the following parameters: LS duration, estimated blood loss (EBL), number of dissected PLNs, and perioperative complications (within 4 months after LS). LS duration was defined as the time from skin incision to closure. The EBL and number of dissected PLNs were retrieved from anesthesia records and pathology reports, respectively. Perioperative complications were evaluated using the Common Terminology Criteria for Adverse Events, Version 4.0 or Clavien-Dindo Classification, Version 2.0.

For the statistical analysis, the Wilcoxon test was used to assess the surgical outcomes. The strengths of correlations were assessed using Spearman's correlation coefficient $(\rho)$, and the degrees of correlation were classified as weak $(\rho=0.2-0.4)$, moderate $(\rho=0.4-$ $0.7)$, or strong $(\rho=0.7-1.0)$. A P value of $<0.05$ was regarded as statistically significant. We used JMP software, Version 13 (SAS Institute Inc., Cary, NC, USA) for the statistical analyses.

\section{RESULTS}

Thirty-eight patients who underwent LS for diagnosed EC between February 2015 and October 2017 were studied retrospectively. PLA was performed in 25 cases, and PLN sampling was performed in nine cases. Extended simple hysterectomy and bilateral salpingo-oophorectomy were performed in two cases. A further two cases transitioned to laparotomy because of ureter injury or peritoneal dissemination of carcinoma.

The characteristics of the 25 patients who underwent PLA are summarized in Table 1. These patients had a median age of 56 years and median BMI of 24.2 $\mathrm{kg} / \mathrm{m}^{2}$. Nine had undergone a previous laparotomy procedure. Preoperative histology revealed grades 1 and $2 \mathrm{EC}$ in 23 and two cases, respectively. Regarding comorbidities, nine, eight, three, and one patient presented with obesity, hypertension, diabetes mellitus, and hyperthyroidism, respectively (including duplicates).

The surgical outcomes are summarized in Table 2. Median duration of LS was 367 minutes in total, 348.5 minutes in Operator A, and 378 minutes in Operator B. The total median EBL was $60 \mathrm{ml}$, with respective values of 57 and $60 \mathrm{ml}$ for Operators A and B. The total median number of dissected PLNs was 22, with respective values of 21.5 and 22 for Operators A and B.

To evaluate LS proficiency, we compared the 
number of LS performed by each operator with the LS duration, EBL, and number of dissected PLNs (Figure 1). For both operators, a moderate but non-significant correlation was observed between the number and duration of LS (Operator A: $\rho=-0.543, p=0.105$; Operator $\mathrm{B}: \rho=-0.472, p=0.076$ ). However, the regression lines indicated a decreasing trend in the LS duration. For both operators, the EBL was small and remained stable from the introduction of LS. This parameter did not correlate significantly with the number of LS performed by either operator (Operator A: $\rho=-0.157$, $\mathrm{p}=0.453$; Operator $\mathrm{B}: \rho=-0.177, \mathrm{p}=0.527)$. However, EBL values as high as $650 \mathrm{ml}$ were recorded shortly after the introduction of LS (Operator A, third case; Operator B, second case). These large blood losses were attributed to difficulty with securing the operative field in an obese patient in the former case and to iliac vein injury in the latter case.

Notably, we observed an inter-operator difference in the correlation between the number of LS performed and the number of dissected PLNs. For Operator B, the number of dissected PLNs decreased as the number of LS increased $(\rho=-0.646, p=0.009)$. By contrast, no significant correlations between the number of dissected PLNs and the LS duration were observed for either operator (Operator A: $\rho=-0.085, p=0.815$; Operator $B: \rho=0.353, p=0.197$ ) (Figure 2). Therefore, the reduced duration of LS was attributed to increased

TABLE 1

Patient characteristics

\begin{tabular}{lc}
\hline & Cases $(\mathrm{n}=25)$ \\
\hline Age $($ years $)$, median (range) & $56(42-79)$ \\
BMI $\left(\mathrm{kg} / \mathrm{m}^{2}\right)$, median (range) & $24.2(17.8-32.0)$ \\
Previous laparotomy & 8 \\
Yes & 17 \\
No & \\
Comorbidity & 9 \\
Obesity ( $\geq$ BMI $\left.25 \mathrm{~kg} / \mathrm{m}^{2}\right)$ & 8 \\
Hypertension & 3 \\
Diabetes mellitus & 1 \\
Hyperthyroidism & \\
Grade of histology & 23 \\
Grade 1 & 2 \\
Grade 2
\end{tabular}

BMI: body mass index proficiency with LS because the duration tended to decrease irrespective of the number of dissected PLNs.

The perioperative complications are summarized in Table 3. Perioperative complications occurred in two and six cases performed by Operators A and B, respectively. One complication was serious and involved a Grade 3 perforation of the sigmoid colon in a
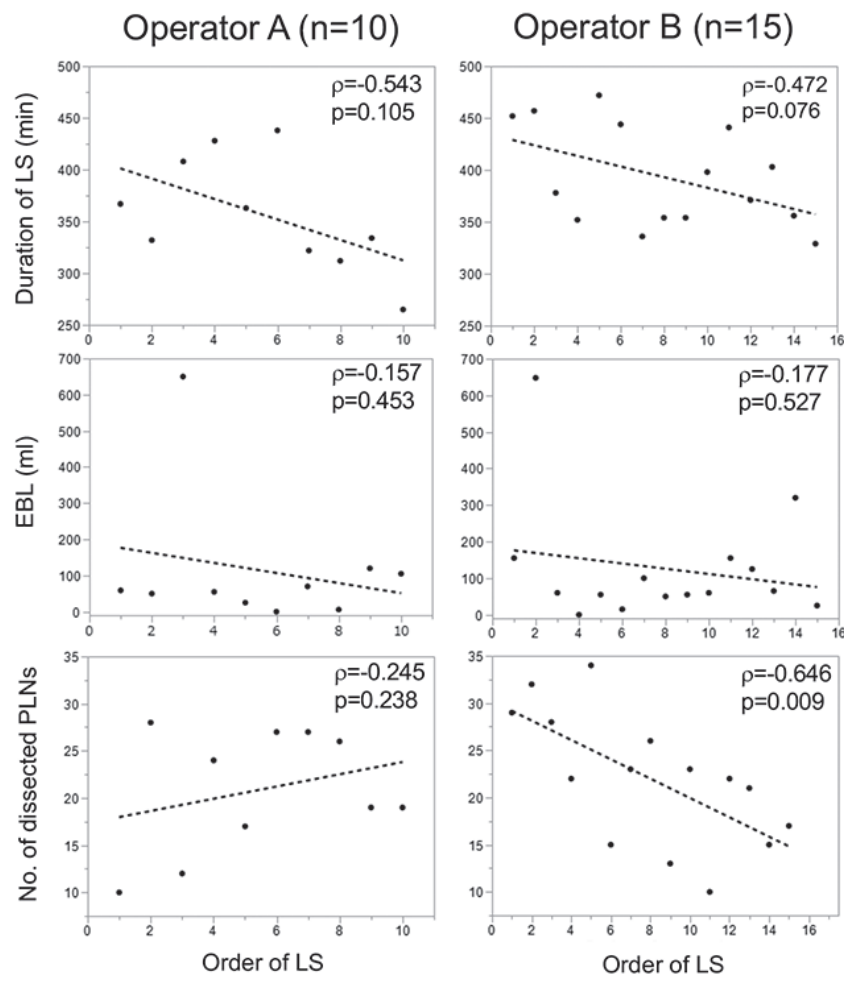

Fig. 1. Duration of laparoscopic surgery (LS), estimated blood loss (EBL), and the number of dissected pelvic lymph nodes (PLNs) according to the number of LS with PLA performed by each operator. The correlations were analyzed using Spearman's correlation coefficient $(\rho)$.

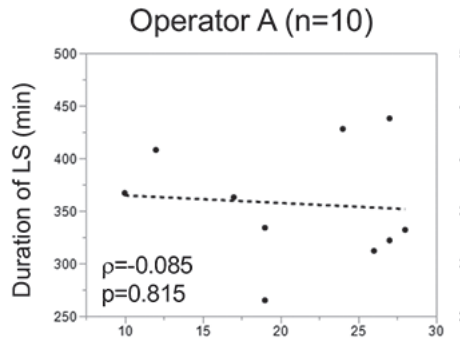

No. of PLNs dissected

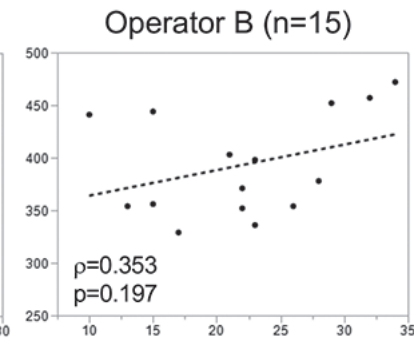

No. of PLNs dissected
Fig. 2. Correlation of the duration of laparoscopic surgery (LS) and the number of pelvic lymph nodes (PLNs) dissected by each operator. The correlations were analyzed using Spearman's correlation coefficient $(\rho)$. 
patient who presented with mild low abdominal pain and fever at 4 days after LS. This patient had an elevated C-reactive protein level of $18.3 \mathrm{mg} / \mathrm{dl}$, and a subsequent computed tomography scan revealed a perforation of the sigmoid colon which was treated via emergent laparotomy. In this case, the manipulation that might have caused perforation of the sigmoid colon could not be determined from the video. However, a preoperative colonoscopy had identified multiple diverticula in the sigmoid colon, and postoperative pathology revealed chronic inflammation around these diverticula. Accordingly, the perforation of the sigmoid colon might be attributed to either the surgical manipulation or incidental diverticulitis, or both.

\section{DISCUSSION}

In this study of two gynecologic oncologists, we observed the development of proficiency after approximately 10 to 15 cases. Regarding safety, only one patient developed a serious complication for which a causal relationship with surgical manipulation could not be confirmed.

Studies of the proficiency of surgery often rely on the duration of the targeted procedure. Although the number of LS cases required for stabilization of the learning curve varied among studies [9-13], in this study, the duration of LS tended to decrease after 10 or 15 cases. We note that although the LS duration tended to increase from the seventh to eleventh cases performed by Operator B, cases 10 and 11 had the second-largest and largest uterine sizes of all cases in this study, and the latter case required an episiotomy for transvaginal removal. Therefore, the actual LS durations in cases 10 and 11 were relatively long (398 and 441 minutes). Notably, the inverse correlation between the number of LS performed and the duration of LS became statistically significant when case 11 was excluded from the analysis $(\mathrm{r}=-0.552, \mathrm{p}=0.041)$.

Table 4 summarizes several studies that investigated the learning curve of LS for EC [9-13]. Notably, the median EBLs of 57 and $60 \mathrm{ml}$ for Operators A and $\mathrm{B}$ in our study were extremely low when compared to those of other studies (115-325 ml) [9-13]. By contrast, Operators A and B dissected considerably higher median numbers of PLNs (21.5 and 22, respectively), compared to other studies (7.8-17.3) [9-13], and this number remained relatively higher even after consid-

TABLE 2.

Surgical outcome

\begin{tabular}{lccc}
\hline & $\begin{array}{c}\text { Total } \\
(\mathrm{n}=25)\end{array}$ & $\begin{array}{c}\text { Operator A } \\
(\mathrm{n}=10)\end{array}$ & $\begin{array}{c}\text { Operator B } \\
(\mathrm{n}=15)\end{array}$ \\
\hline Time (minutes), median (range) & $367(265-472)$ & $348.5(265-438)$ & $378(329-472)$ \\
EBL (ml), median (range) & $60(0-650)$ & $57(0-650)$ & $60(0-648)$ \\
No. of PLN dissected, median (range) & $22(10-34)$ & $21.5(10-28)$ & $22(10-34)$ \\
\hline
\end{tabular}

EBL: Estimated blood loss, PLN: pelvic lymph node,

TABLE 3.

Perioperative complications

\begin{tabular}{lccc}
\hline & \multicolumn{2}{c}{ Numbers in chronological order of LS } & \\
\cline { 2 - 3 } Complications & Operator A $(\mathrm{n}=10)$ & Operator B (n=15) & No. of cases \\
\hline Peripheral sensory neuropathy (G1) & $\# 1,10$ & 2 \\
Injury of vessels (G1) & $\# 2$ & $\# 3, \# 4$ & 1 \\
Lymphocele (G1) & $\# 6$ & $\# 6$ & 2 \\
Lymphedema (G2) & & 1 \\
Chylous ascites (G2) & $\# 7$ & 6 & 1 \\
Perforation of colon (G3) & 2 & 8 \\
\hline Total number of cases & & 1 \\
\hline
\end{tabular}


ering the decrease in the number of dissected PLNs by Operator B with increasing experience. Potentially, a high level of experience with laparotomy for EC contributed to the small EBL volumes and relatively large number of dissected PLNs recorded for the operators in our study.

As noted above, a severe complication (sigmoid colon perforation) was observed in only one case. Although it was difficult to determine whether surgical manipulation or diverticulitis was the cause, this outcome underscores the importance of careful intestinal tract manipulation. Additionally, this study was limited by the retrospective design, single institution setting, and small number of cases. Potentially, a further increase in the number of LS cases may lead to further reductions in operation times and a shift in the number of cases necessary to stabilize the learning curve.

\section{CONCLUSION}

The achievement of proficiency with LS requires knowledge of anatomy, experience with laparotomy, and training in laparoscopic handling. Gynecologic oncologists may develop LS proficiency in a relatively shorter period, as they already possess the requisite anatomical knowledge and tend to have some experience with laparotomy.

CONFLICT OF INTERESTS: The authors declare that they have no competing interests.

ACKNOWLEDGEMENT: This work was supported in part by the Supporting Fund of Obstetrics and Gynecology of Kurume University.

TABLE 4.

Summary of studies on learning curve of laparoscopic surgery for endometrial cancer

\begin{tabular}{|c|c|c|c|c|c|}
\hline First author, year & $\begin{array}{c}\text { Eltabbakh GH } \\
2000\end{array}$ & $\begin{array}{c}\text { Holub Z } \\
2003\end{array}$ & $\begin{array}{c}\operatorname{Lim}_{2011} \mathrm{PC} \\
\text {. }\end{array}$ & $\begin{array}{c}\text { Rad MT } \\
2013\end{array}$ & $\begin{array}{c}\text { Bennich G } \\
2016\end{array}$ \\
\hline No. of cases & 75 & 108 & 122 & 20 & 49 \\
\hline Type of surgery & LAVH & LAVH & TLH & TLH & TLH \\
\hline \multirow{5}{*}{$\begin{array}{l}\text { Duration of surgery } \\
\text { (minutes) } \\
\text { phase: average }\end{array}$} & Ph1 231.8* & Ph1 156.3* & Ph1 196.7 & Ph1 397.7* & Ph1 $180^{*}$ \\
\hline & $\mathrm{Ph} 2$ 175.9* & $\mathrm{Ph} 2$ 148.8* & $\mathrm{Ph} 2180.4$ & $\mathrm{Ph} 2300.6^{*}$ & Ph2 $153^{*}$ \\
\hline & Ph3 167.7* & Ph3 142.8* & & & Ph3 130* \\
\hline & & & & & $\mathrm{Ph} 4115^{*}$ \\
\hline & & & & & $\mathrm{Ph} 590^{*}$ \\
\hline \multirow{3}{*}{$\begin{array}{l}\text { EBL }(\mathrm{ml}) \\
\text { Phase: average }\end{array}$} & Ph1 325 & Ph1 240 & Ph1 220.8 & Ph1 222.2 & NA \\
\hline & $\mathrm{Ph} 2243$ & $\mathrm{Ph} 2232$ & $\mathrm{Ph} 2198.6$ & $\mathrm{Ph} 279.5$ & \\
\hline & Ph3 278 & Ph3 224 & & & \\
\hline \multirow{5}{*}{$\begin{array}{l}\text { No. of PLN } \\
\text { Phase: average }\end{array}$} & $\mathrm{Ph} 17.8^{*}$ & Ph1 $12.4^{*}$ & Ph1 23.2 & Ph1 17.3 & Ph1 $14^{*}$ \\
\hline & $\mathrm{Ph} 210.6^{*}$ & $\mathrm{Ph} 213.9^{*}$ & $\mathrm{Ph} 225.7$ & $\mathrm{Ph} 222.5$ & $\mathrm{Ph} 214^{*}$ \\
\hline & $\operatorname{Ph} 311.9^{*}$ & $\mathrm{Ph} 315.4^{*}$ & & & $\mathrm{Ph} 317^{*}$ \\
\hline & & & & & $\mathrm{Ph} 420^{*}$ \\
\hline & & & & & $\operatorname{Ph} 527^{*}$ \\
\hline $\begin{array}{l}\text { Transition to } \\
\text { laparotomy }\end{array}$ & 2 & 5 & & 3 & \\
\hline $\begin{array}{l}\text { No. of cases for LC } \\
\text { stability }\end{array}$ & 25 & $25-30$ & & 9 & $>49$ \\
\hline
\end{tabular}

LAVH: laparoscopic assisted hysterectomy, TLH: total laparoscopic hysterectomy, Ph: Phase, EBL: estimated blood loss, NA: not available, PLN: pelvic lymph node, LC: learning curve.

* showed statistically significant difference 


\section{REFERENCES}

1. Common Registry and Statistics. Cancer informative Service, National Cancer Center, Japan.

2. Yamagami W, Nagase S, Takahashi F, Ino K, Hachisuga T et al. Clinical statistics of gynecologic cancers in Japan. J Gynecol Oncol 2017; 28(2):e32.

3. Wright JD, Burke WM, Tergas AI, Hou JY, Huang Y et al. Comparative effectiveness of minimally invasive hysterectomy for endometrial cancer. J Clin Oncol 2016; 34(10):1087-1096.

4. Obermair A, Janda M, Baker J, KondalsamyChennakesavan S, Brand A et al. Improved surgical safety after laparoscopic compared to open surgery for apparent early stage endometrial cancer: results from a randomised controlled trial. Eur J Cancer 2012; 48(8):1147-1153.

5. Mourits MJ, Bijen CB, Arts HJ, ter Brugge HG, van der Sijde R et al. Safety of laparoscopy versus laparotomy in early-stage endometrial cancer: a randomised trial. Lancet Oncol 2010; 11(8):763-771.

6. Walker JL, Piedmonte MR, Spirtos NM, Eisenkop SM, Schlaerth JB et al. Laparoscopy compared with laparotomy for comprehensive surgical staging of uterine cancer: Gynecologic Oncology Group Study LAP2. J Clin Oncol 2009; 27(32):5331-5336.

7. Janda M, Gebski V, Davies LC, Forder P, Brand A et al. Effect of total laparoscopic hysterectomy vs total abdominal hysterectomy on disease-free survival among women with stage I endometrial cancer: a randomized clinical trial.
JAMA 2017; 317(12):1224-1233.

8. Mota F, Vergote I, Trimbos JB, Amant F, Siddiqui N et al. Classification of radical hysterectomy adopted by the gynecological cancer group of the European organization for research and treatment of cancer. Int J Cancer 2008; 18(5):1136-1138.

9. Eltabbakh GH. Effect of surgeon's experience on the surgical outcome of laparoscopic surgery for women with endometrial cancer. Gynecol Oncol 2000; 78(1):58-61.

10. Holub Z, Jabor A, Bartos P, Hendl J, and Urbánek S. Laparoscopic surgery in women with endometrial cancer: the learning curve. Eur J Obstet Gynecol Reprod Biol 2003; 107(2):195-200.

11. Tahmasbi Rad M, Wallwiener M, Rom J, Sohn C, and Eichbaum M. Learning curve for laparoscopic staging of early and locally advanced cervical and endometrial cancer. Arch Gynecol Obstet 2013; 288(3):635-642.

12. Bennich G, Rudnicki M, and Lassen PD. Laparoscopic surgery for early endometrial cancer. Acta Obstet Gynecol Scand 2016; 95(8):894-900.

13. Lim PC, Kang E, and Park DH. A comparative detail analysis of the learning curve and surgical outcome for robotic hysterectomy with lymphadenectomy versus laparoscopic hysterectomy with lymphadenectomy in treatment of endometrial cancer: A case-matched controlled study of the first one hundred twenty two patients. Gynecol Oncol 2011; 120(3):413-418. 